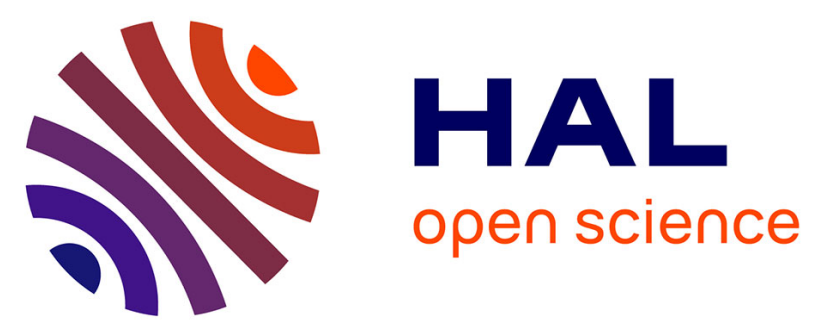

\title{
Laparoscopic optical biopsies: In vivo robotized mosaicing with probe-based confocal endomicroscopy
}

Benoît Rosa, Benoît Herman, Jérome Szewczyk, Brice Gayet, Guillaume Morel

\section{To cite this version:}

Benoît Rosa, Benoît Herman, Jérome Szewczyk, Brice Gayet, Guillaume Morel. Laparoscopic optical biopsies: In vivo robotized mosaicing with probe-based confocal endomicroscopy. 2011 IEEE/RSJ International Conference on Intelligent Robots and Systems (IROS 2011), Sep 2011, San Francisco, United States. 10.1109/IROS.2011.6094513 . hal-01765044

\section{HAL Id: hal-01765044 \\ https://hal.science/hal-01765044}

Submitted on 12 Apr 2018

HAL is a multi-disciplinary open access archive for the deposit and dissemination of scientific research documents, whether they are published or not. The documents may come from teaching and research institutions in France or abroad, or from public or private research centers.
L'archive ouverte pluridisciplinaire HAL, est destinée au dépôt et à la diffusion de documents scientifiques de niveau recherche, publiés ou non, émanant des établissements d'enseignement et de recherche français ou étrangers, des laboratoires publics ou privés. 


\title{
Laparoscopic Optical Biopsies: In Vivo Robotized Mosaicing with Probe-based Confocal Endomicroscopy
}

\author{
Benoît Rosa, Benoît Herman, Jérôme Szewczyk, Brice Gayet and Guillaume Morel
}

\begin{abstract}
Probe-based confocal laser endomicroscopy is a promising technology for performing minimally-invasive optical biopsies. With the help of mosaicing algorithms, several studies reported successful results in endoluminal surgery. In this paper, we present a prototype for making robotized optical biopsies on a variety of organs inside the abdominal cavity. We chose a macro-micro association, with a macropositioner, a micropositioner and a passive mechanical compensation of physiological motion. The probe is actuated by three hydraulic micro-balloons and can be moved on the surface of an organ to generate a mosaic. This paper presents the design and experimental results of a first in vivo trial on a porcine model.
\end{abstract}

\section{INTRODUCTION}

Probe-based confocal laser endomicroscopy allows to grab videos in vivo and in situ of living tissues in real time with very high resolution. The Cellvizio from Mauna Kea Technologies (Paris, France), used along with a Gastroflex UHD probe, provides images at 12 frames/s with a $1 \mu \mathrm{m}$ lateral resolution and $240 \mu \mathrm{m} \times 200 \mu \mathrm{m}$ field of view. Although the field of view is too narrow to be used for anatomopathological analysis, there is a possibility of sweeping the organ surface with the probe and reconstructing, from the recorded images, a wide mosaic offering superresolution [1], [2]. Several studies reported its success in endoluminal examinations [3]-[5].

Our aim is to perform optical biopsies on the organs inside the abdominal cavity via a laparoscopic access, in order to determine if cancer patients are appropriate candidates for surgery or would be better suited to chemotherapy or radiation treatment. In most cases, the standard protocol is to take a tissue sample-a biopsy-at the beginning of the surgical procedure and to have it analyzed by an anatomopathologist under a traditional microscope. This is invasive and time consuming, especially since the biopsy must be frozen and cut before the analyst is able to put it under the microscope. Alternatively, proceeding to optical biopsies during a minimally-invasive diagnostic procedure could decrease the risks related to inappropriate curative surgery. A minimally invasive laparoscopic device that can bring the probe in contact of a tissue and actuate

Authors are with UPMC Univ Paris 06, UMR 7222, ISIR, F-75005, Paris, France and CNRS, UMR 7222, ISIR, F-75005, Paris, France \{rosa, szewczyk, morel\}@isir.upmc.fr

B. Herman is now F.R.S.-FNRS Postdoctoral Researcher with Université catholique de Louvain, Center for Research in Mechatronics, B-1348 Louvain-la-Neuve, Belgium benoit.herman@uclouvain.be

B. Gayet is also with Université Paris Descartes and the Department of Digestive Diseases, Montsouris Institute, 42 Boulevard Jourdan, 75014 Paris, France it to perform the optical biopsy is here presented. The system is composed of three parts : a macropositioner, a micropositioner and a stabilizer.

The function of the macropositioner is to carry out the large displacements that are needed to find the zones to biopsy, under direct control of the surgeon. It must bring the micropositionner orthogonal to the surface to biopsy. Such a system was previsouly designed by Rouse et al. in [6]. However, this prototype, similar to a conventional laparoscope, was dedicated to ovarian tissue, and the insertion point could be chosen to get the probe orthogonal to the tissues of this organ. Therefore, this system seems not to be suitable for our application, and distal degrees of freedom seem to be mandatory for the macropositioner. The i-Snake is a snake-like endoscope designed to overcome such difficulties [7]. However, its too numerous inner degrees of freedom make it complex to control, and its size- $12 \mathrm{~mm}$ outer diameter with only one $3 \mathrm{~mm}$ channel-is too large with regard to minimal invasiveness constraints. Rather, we used a prototype of bendable laparoscope with $6 \mathrm{~mm}$ outer diameter. The internal degrees of freedom were then limited to two.

We aim at making optical biopsies with a surface of approximately $3 \mathrm{~mm}^{2}$. Though fair quality mosaics can be obtained with manual actuation of the probe [1], [2], quality of the images and mosaics is very sensitive to the speed of movements along the tissue surface. Therefore, a manual actuation of the probe, where the speed and direction of the movements are not controlled, often leads to low quality images. Controlling the trajectory and the speed of the probe can lead to better quality mosaics and therefore a better analysis by the pathologists. For these reasons we propose a robotized micropositioning device, with a predefined scanning trajectory of the tissues to get a constant shape mosaic with a suitable speed.

Finally, organs in the abdominal cavity are not fully constrained and move with breathing and peristalsis. These movements have a typical frequency ranging generally from 0.2 to $1 \mathrm{~Hz}$, and can be of several centimeters of magnitude. This leads to a very unstable image and, if any, a bad quality mosaic with uncontrolled shape. Fig. 1 shows six images taken in vivo on a porcine model with a confocal probe at the contact of the liver. The probe was put at the contact of the organ with a standard laparoscopic instrument, and was not actuated. The images are very unstable and cannot be 


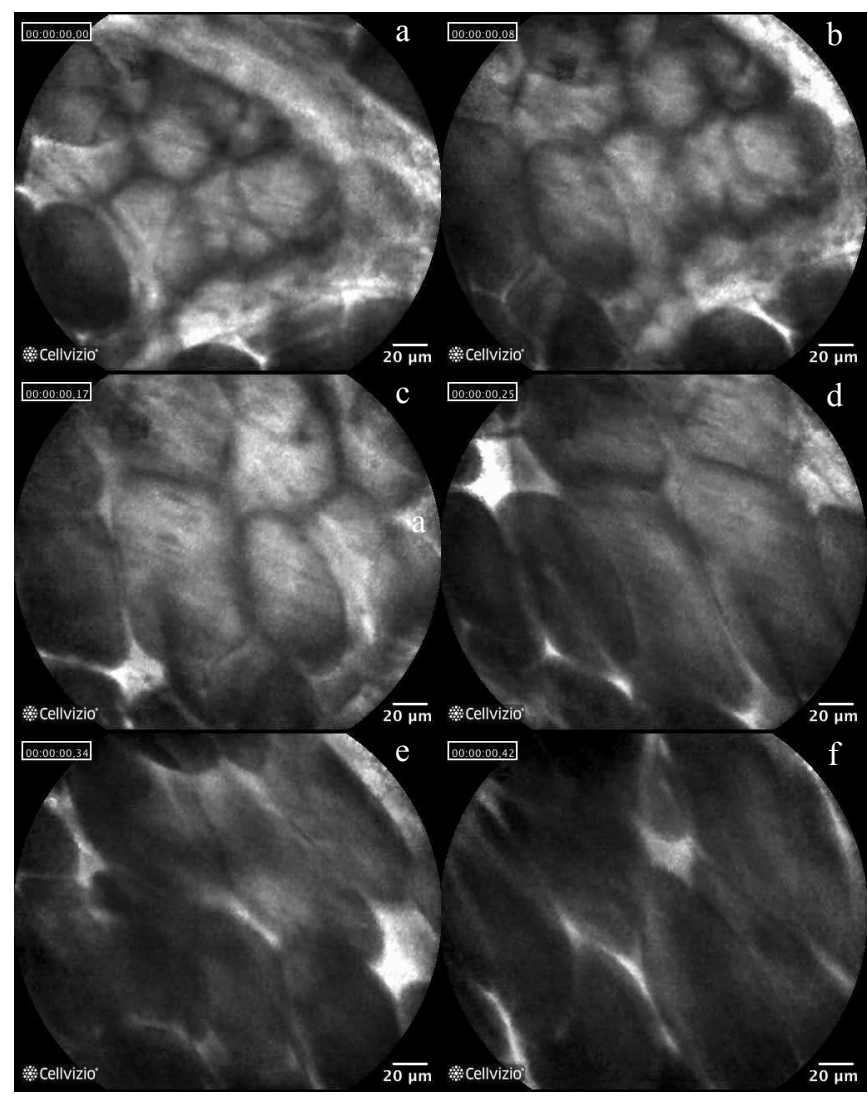

Fig. 1. Confocal images taken in vivo on the liver of a porcine model with a Gastroflex UHD probe, without stabilization: (a) $t_{0}$; (b) $t_{0}+0.08 \mathrm{~s}$; (c) $t_{0}+0.17 \mathrm{~s}$; (d) $t_{0}+0.25 \mathrm{~s}$; (e) $t_{0}+0.34 \mathrm{~s}$; (f) $t_{0}+0.42 \mathrm{~s}$

processed by the mosaicing algorithm.

To solve this problem, Lee et al. proposed a physiological motion compensation method based on visual servoing and a piezoelectric actuator at microscopic scale [8]. The motion compensated images had a residual motion of less than $20 \mu \mathrm{m}$ which, compared to the probe field of view $(200 \times 240 \mu \mathrm{m})$, is satisfactory. However, the subject was a mouse and a mechanical stabilizer was used to reduce the physiological motion prior to software stabilization. Hence, the movements to compensate had a magnitude of $150 \mu \mathrm{m}$ only-about 100 times smaller than the movements observed in the abdomen of human beings. Finally, the size of the piezoelectric actuator makes it rather difficult to transpose the design in the abdominal cavity. In [9], Ott et al. also presented a visual servoing compensation algorithm, based on a predictive model. However, the precision required by our application is too high for using such a system. Closer to our problem, Noonan et al. proposed in [10] a force adaptive model to keep a constant interaction force between the probe and the tissue. However, organs move in 3D in the abdominal cavity, while this system only has one force-controlled degree of freedom. In this paper, we propose a passive mechanical compensation that is described below.

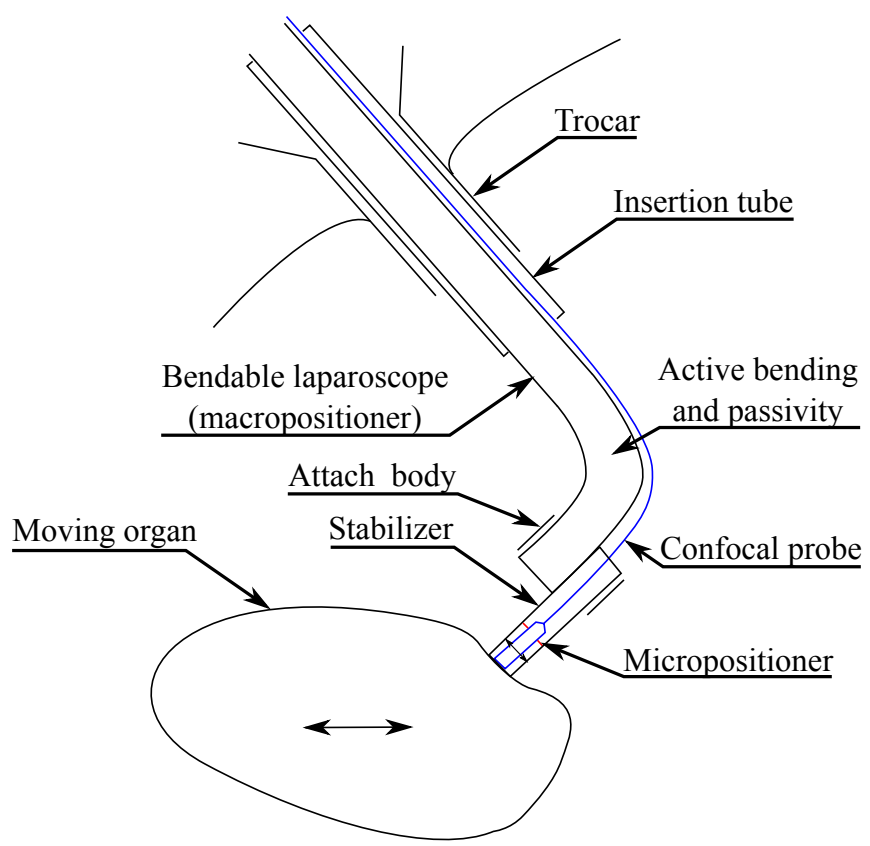

Fig. 2. Schematic view of the system. Note that the active bending is realized with external motors and cables

\section{PRototype Description}

The system that we propose consists in a macropositioner that brings the optical probe on the surface of the tissue to biopsy, a mechanical stabilizer mounted on the macropositioner tip, and a micropositioner inside the stabilizer that moves the probe to perform a mosaic (see Fig. 2). The system is inserted in the abdominal cavity through a $12 \mathrm{~mm}$ diameter trocar. The macropositioner embeds a CCD camera and two fiber optic light guides to see inside the patient's body, as in a standard laparoscopic procedure. The macropositioner also comprises an outer insertion tube with several hermetic channels used to insert the probe and the micropositioner distal actuators, as detailed below.

\section{A. Macropositioner}

The two functions that the macropositioner carries out are the large movements required for the exploration of the abdominal cavity, and the positioning of the micropositionner, holding the probe, normally to the tissue. We use a prototype of bendable laparoscope with $6 \mathrm{~mm}$ outer diameter. It integrates a CCD chip and two fiber optic light guides at its tip. It does not posses any additional operating channel. The laparoscope can bend in two directions, from -90 to +90 degrees each.

Rigid laparoscopes are commonly used in standard laparoscopic procedures for accessing a large variety of organs in the abdominal cavity. In our case, the general exploration is possible using this conventional technique, while the two degrees of freedom of the laparoscope are used for orienting its tip (see Fig. 3), in order to position the micropositioner normally to the tissue surface.

The global positioning of the macropositioner can be performed by direct manual guidance of the surgeon, whereas 
the two internal degrees of freedom are motorized. Two external motors equipped with a pulley tighten two pairs of opposing cables that run through the laparoscope and are attached to its tip, like most fiberscopes. In our first prototype, they are controlled using a foot switch. This allows the surgeon to keep one hand free while manipulating the macropositioner, for instance if an instrument (e.g. a grasper) is required to manipulate organs. To help the surgeon stabilize the device while performing a microscan, the macropositioner can be immobilized using a table-mounted lockable arm.

\section{B. Passive Compensation of Physiological Motion}

As explained in Section I above, standard compensation schemes have major drawbacks that make them unsuitable for our application. To circumvent these limitations, we chose a passive mechanical stabilization. By attaching a $5.45 \mathrm{~mm}$ outer diameter circular tube on the laparoscope distal tip (see the part labelled "stabilizer" in Fig. 2), a pressure can be applied on the organ to keep a stable contact with it despite its motion. The outer diameter of the stabilizer is chosen to ensure that the whole systemincluding the $6 \mathrm{~mm}$ laparoscope-fits a standard $12 \mathrm{~mm}$ trocar. Some flexibility is added between the insertion point in the abdominal wall and the stabilizer, so that the distal tip can follow the organ motion passively instead of restricting it (in opposition to beating heart stabilization). The bending part of the macropositioner exhibits enough flexibility to ensure this function: stiffness ranges from 0.76 to $1.29 \mathrm{~N} / \mathrm{rad}$ at the distal end of the tip, depending on the bending angle.

Our motion compensation principle has three advantages. Firstly, it works passively and does not require any complex control and actuation scheme. Secondly, the flexible part in the structure also filters small unwanted movements of the macropositionner (e.g. surgeon's hand tremor, insertion point motion due to breathing or insufflation). Finally, we can use

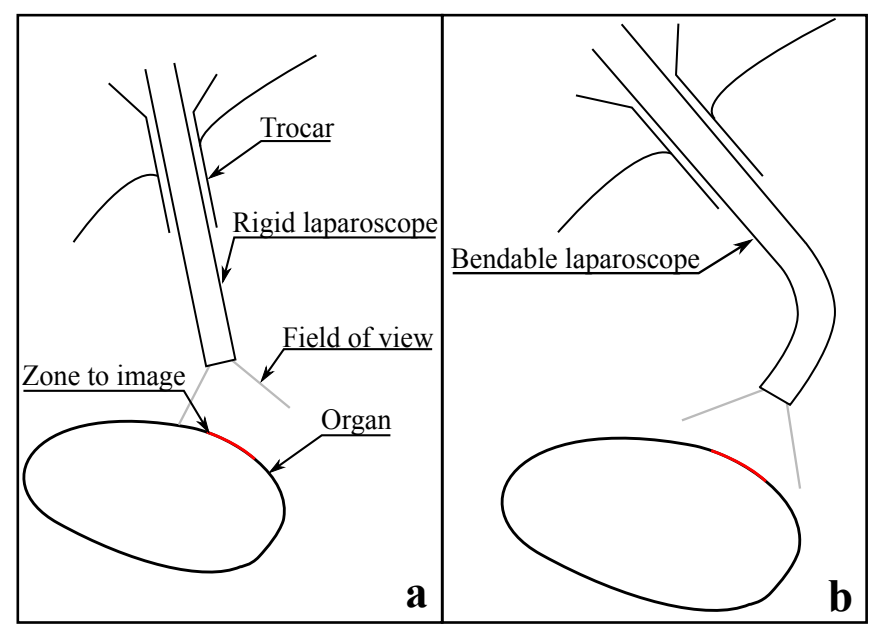

Fig. 3. Comparison of standard rigid laparoscope (a) and bendable laparoscope (b) for imaging a zone on an organ with a fixed incision. For sole exploration purposes, the standard laparoscope may be sufficient, however the two internal degrees of freedom allow the surgeon to position the bendable laparoscope normally to the tissue it as a reference for the micropositioning, having therefore an appropriate control of the confocal probe motion with respect to the tissue to biopsy. This is detailed hereafter.

\section{Micropositioner}

1) Integration: The micropositioner is a miniature device that is in charge of moving the endomicroscopic laser probe along a trajectory so as to acquire images that will be merged afterwards by the mosaicing algorithm. The confocal probe consists of a flexible bundle of optical fibers (outer diameter: $1.4 \mathrm{~mm}$ ) at the tip of which an optical head hosting the micro lenses is mounted. The optical head is a $12 \mathrm{~mm}$ long cylinder, with an outer diameter equal to $2.6 \mathrm{~mm}$.

Images must satisfy several constraints in order to be processed properly. The movement must be slow and continuous on the $3 \mathrm{~mm}^{2}$ zone, which can be circular or square. In fact, the scanning speed of the laser spot in the confocal microscope [1] imposes a maximal speed along the surface of $1 \mathrm{~mm} / \mathrm{s}$. Moreover, the mosaicing algorithm needs a minimum overlapping of $30 \%$ of the image to function properly. The UHD probe has a $240 \mu \mathrm{m} \times 200 \mu \mathrm{m}$ field of view, so the total time required to cover a square mosaic of $3 \mathrm{~mm}^{2}$ at $1 \mathrm{~mm} / \mathrm{s}$ constant speed with $30 \%$ of overlapping will be about $21 \mathrm{~s}$.

As depicted in Fig. 2, the micropositioner is located inside the stabilizer tube and uses it as a reference. Another design constraint is therefore that the micropositioner has a $5 \mathrm{~mm}$ diameter-equal to the inner diameter of the tubeincluding the probe and actuation means.

We chose hydraulic actuation, using sterile balloon catheters. The catheters diameter is $0.9 \mathrm{~mm}$, and the balloons maximal diameter when inflated is $4 \mathrm{~mm}$. Fig. 4 shows the actuation principle used for obtaining a one degree of freedom motion. NiTi superelastic wires are used for keeping

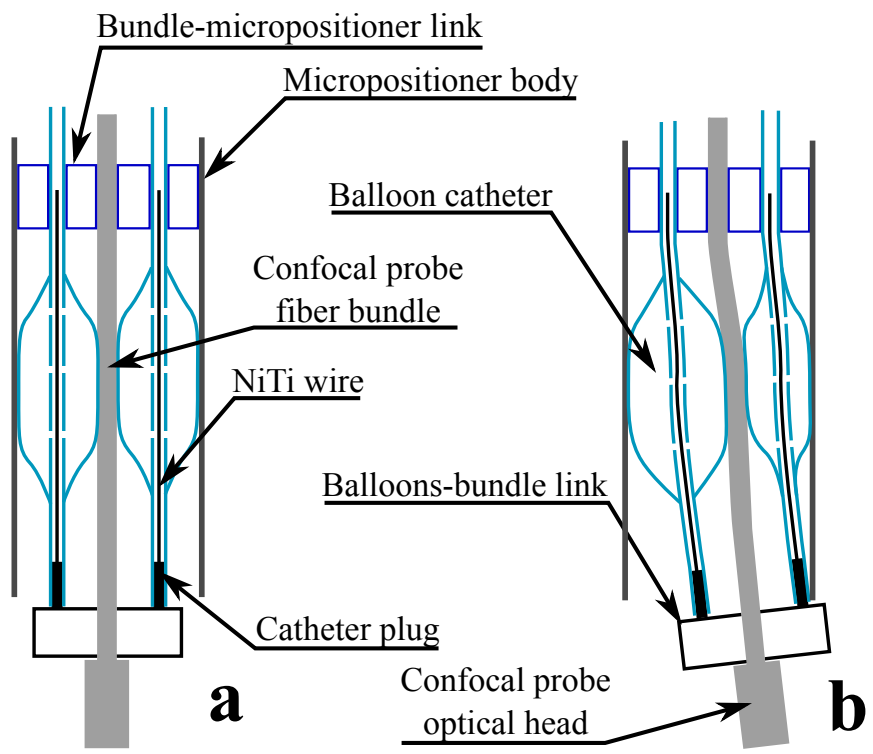

Fig. 4. Actuation principle with two balloon catheters. a: the two balloons have the same volume, the probe is at the center of the micropositioner. $b$ : one balloon is over-inflated and the other is under-inflated. 
the ballons straight while inflated, and the catheter plugs are used to close it for preventing water leakage.

As shown in Fig. 5, three balloons are mounted at 120 degrees around the fiber bundle to obtain a nearly planar motion, similar to the prototype of Ruzzu et al. in [11]. On the contrary of their prototype however, the use of hydraulic actuation instead of pneumatic actuation brings several advantages: improved safety (neither electrical control of the valves nor thermal heating), control of the inflation and the deflation of the balloons using the volume of fluid injected. For the latter, assuming that the water is incompressible, the inflated volume is directly linked to the diameter of the balloon, and therefore to the probe displacement.

2) Motion control: The balloons inflation is controlled by syringes outside the patient's body, filled with water and plugged onto three precision linear motorized tables (see Fig. 6). Our control variables are the volumes of the three balloons $V_{1}, V_{2}$ and $V_{3}$.

Denoting $V_{\max }$ the maximum volume in the balloons, it was first observed that choosing:

$$
V_{1}=V_{2}=V_{3}=\frac{2 V_{\max }}{3}
$$

led in centering the probe in the middle of its workspace, with a satisfactory rigidity. From this rest position, increasing simultaneously the volumes of the three balloons, would result in no motion and an increased stiffness, due to symmetry. Providing a motion can rather be obtained by increasing the volume injected in one or two balloons while decreasing the volume of two or one balloon. To make it more clear, consider the schematic representation of Fig. 7. Starting from the rest position, a small displacement $\Delta x, \Delta y$ can be expressed from a small variation $\Delta V_{i}, i \in\{1, . ., 3\}$ of the three volumes by:

$$
\left(\begin{array}{c}
\Delta x \\
\Delta y
\end{array}\right)=J(x, y)\left(\begin{array}{l}
\Delta V_{1} \\
\Delta V_{2} \\
\Delta V_{3}
\end{array}\right),
$$

where $J$ is the jacobian matrix of the system. Formal derivation of $J$ and its inversion under the constraint:

$$
f\left(V_{1}, V_{2}, V_{3}\right)=0,
$$

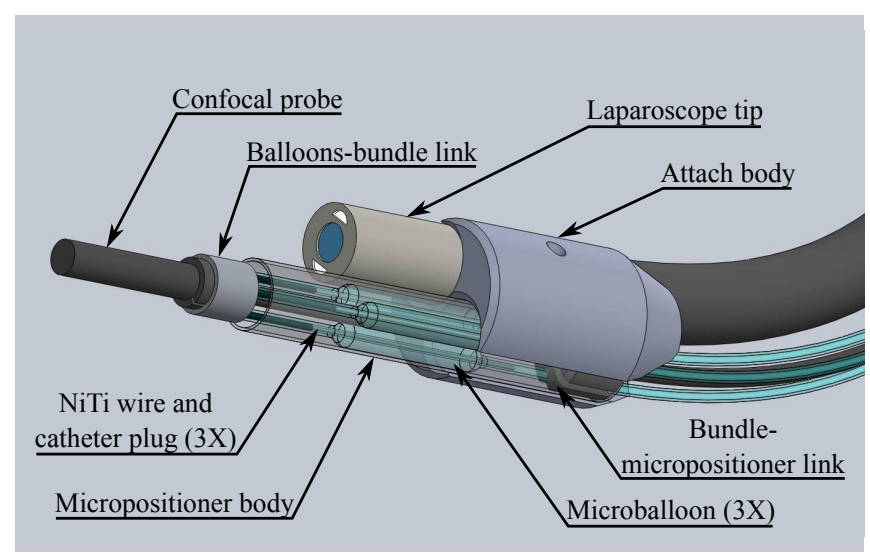

Fig. 5. CAD view of the micropositionner

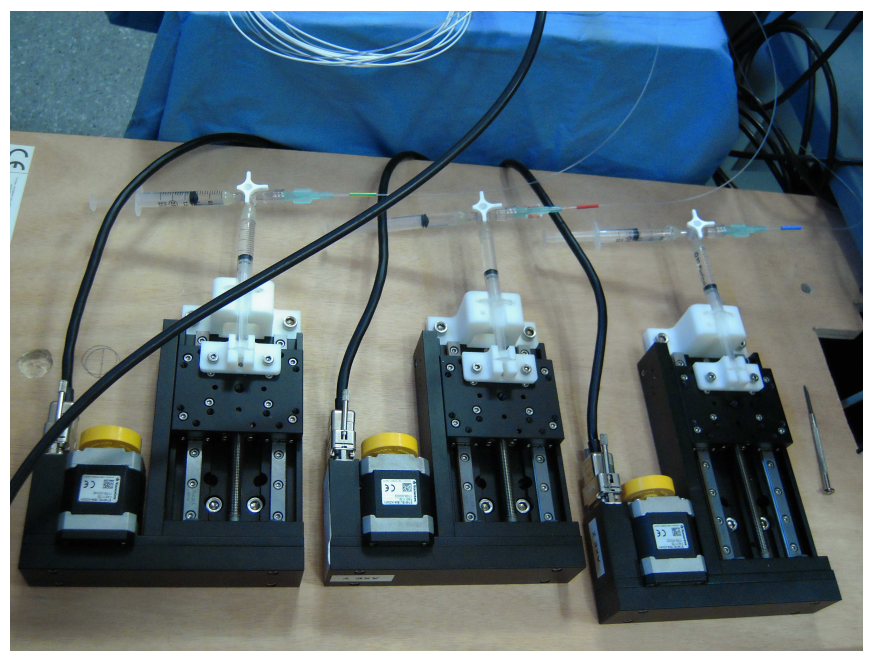

Fig. 6. Inflation of the balloons is controlled by three syringes mounted on precision linear actuators

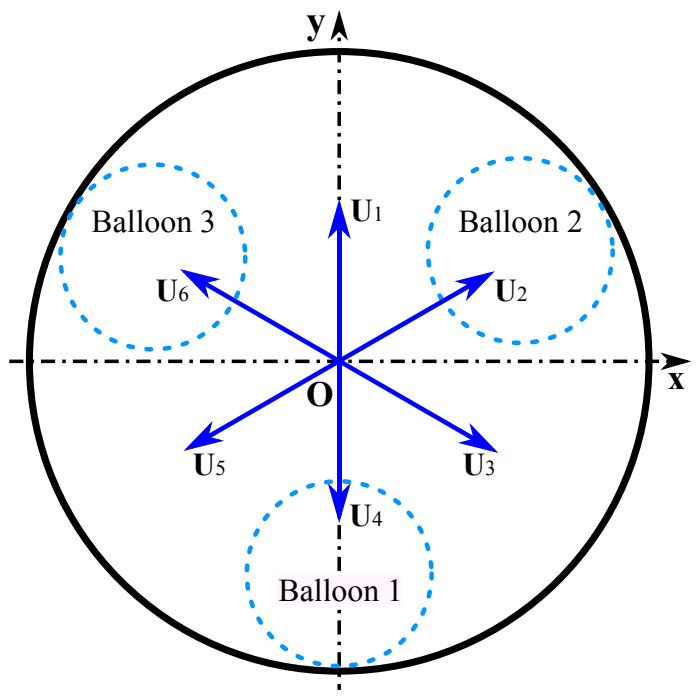

Fig. 7. By inflating one balloon and deflating symmetrically the two others, the six directions $\left\{\overrightarrow{u_{1}}, \overrightarrow{u_{2}}, \overrightarrow{u_{3}}, \overrightarrow{u_{4}}, \overrightarrow{u_{5}}, \overrightarrow{u_{6}}\right\}$ can be reached

used to express the fact that the total volume should be enough to maintain the contact with the probe and enough rigidity, is rather complex and behind the scope of our study.

In a much simpler way, an open loop controller was developed. Consider again the schematic representation of Fig. 7. It can be seen, and was experimentally verified, that, choosing:

$$
\left(\Delta V_{1}, \Delta V_{2}, \Delta V_{3}\right)=(\Delta V,-\Delta V,-\Delta V),
$$

with $\Delta V$ a small positive volume, produces a motion of the probe along the direction $U_{1}$. Similarly, canonic variations of $\Delta V_{i}$ can produce motions along $U_{2}, U_{3}, \ldots, U_{6}$.

Therefore, an open loop controller with 6 separate discrete controls was developed. The probe can be moved along one of the six directions using six canonical patterns for the volume variation. Of course, this open loop controller is not very accurate, but it appeared to be enough for the first 


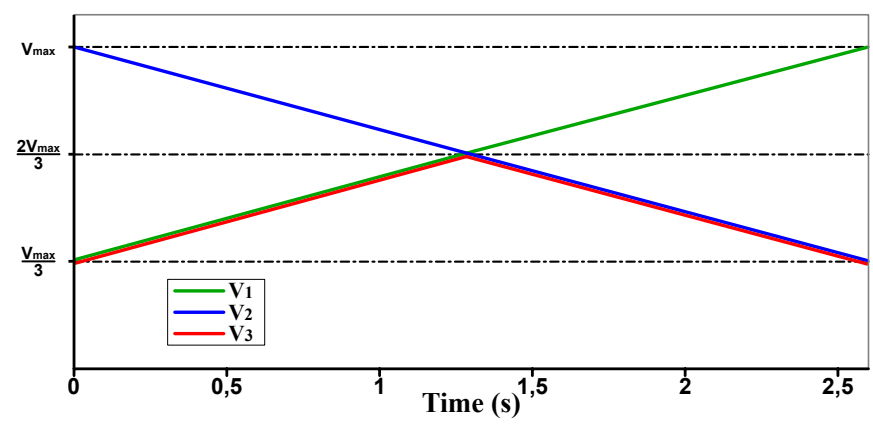

Fig. 8. Volumes of the balloons during the freespace trial. $V_{\max }=$ $0.15 \mathrm{~mL}$

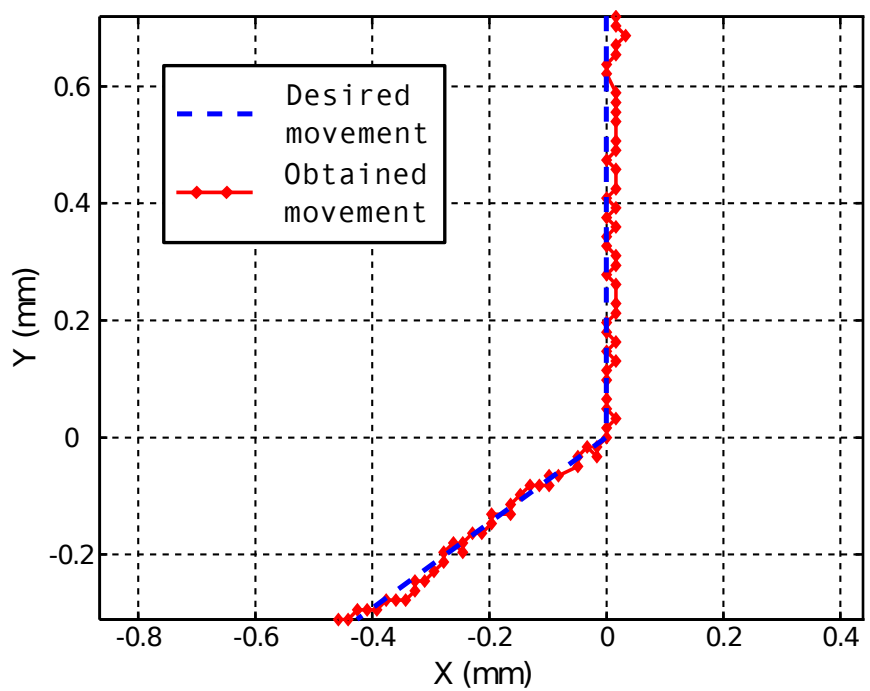

Fig. 9. XY graph of the freespace movement during a trial. The positions were taken from images at a constant framerate of 30 images/s

experiments. Indeed, the aim of this first study was to show that an actuation with hydraulic balloons was possible, with enough smoothness on the probe speed to obtain mosaics. Further studies will involve a better modeling of the system, along with a closed-loop cinematic control of the whole system.

The control of the system was done with a computer controlling the movement of the linear actuators presented on Fig. 6. Six buttons control each one of the directions $U_{1}$ to $U_{6}$. Because the system is controlled with an approximated law, drift in the position is very likely to occur. For this reason, a seventh button was added to bring the micropositioner back at the rest position $\left(V_{1}=V_{2}=V_{3}=\frac{2 V_{\max }}{3}\right)$.

\section{EXPERIMENTAL TRIALS}

The micropositioning system was first tested ex vivo in free space to assess idle stroke and precision of position and speed during motions on a predefined trajectory. The complete system with macropositionner was then tested in vivo on a porcine model.

\section{A. Free space tests}

The prototype was tightened on a workbench. A calibrated $30 \mathrm{frames} / \mathrm{s}$ camera placed in front of the micropositionner

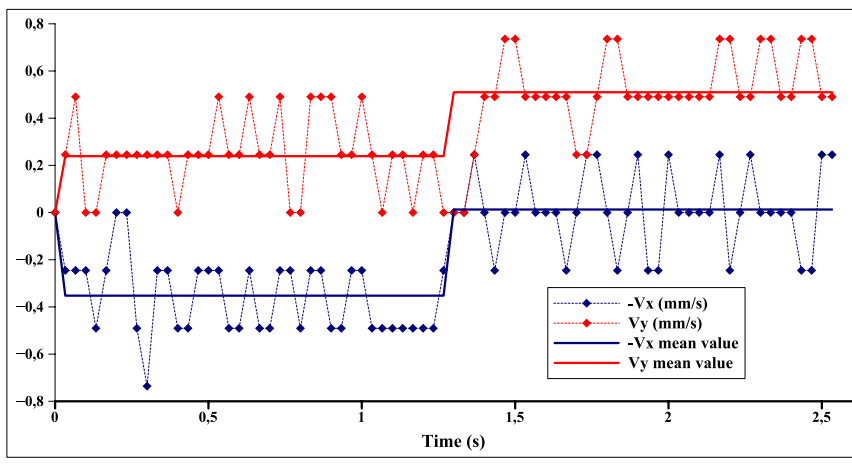

Fig. 10. Speed of the probe tip during the freespace trial. $-\mathrm{Vx}$ is here presented instead of $\mathrm{Vx}$ for clarity reasons

was used to record the motions of the probe tip. The trial consists in moving the probe at constant speed along a path made of two straight lines. These lines intersect at the center of the micropositionner and make an angle of 120 degrees. The volumes controlled in the three balloons during the trial are shown in Fig. 8.

Fig. 9 shows the XY coordinates of the probe tip during the trial. One can see that the direction is controlled accurately and that the speed is rather regular. The average error in position is $17.8 \mu \mathrm{m}$ (standard deviation: $12.8 \mu \mathrm{m}$ ) and the maximum error is $50.6 \mu \mathrm{m}$, which are the tenth and quarter of the probe field of view, respectively. As foreseen, control over the XY position of the probe tip is accurate along the preferred directions defined previously.

Fig. 10 shows the speed of the probe tip during the trial. Due to the fact that the probe moves slowly and with little magnitude, the measures present a lot of noise that is mainly due to quantification (desired speed equals 1 pixel per timeframe). Fig. 10 also presents the speed mean value, which is in accordance with the results of Fig. 9. In the next section, in vivo trials involving contacts between the probe tip and the organs are presented.

\section{B. In Vivo Validation}

The prototype was tested on a porcine model by an experienced surgeon (Fig. 11). The pig was under global anesthesia and $10 \mathrm{ml}$ of fluoresceine (1\% dilution) were injected as a fluoroscopy agent for confocal microscopy. A lockable arm was mounted on a table lateral rail to hold the macropositioner. The system was introduced in the abdominal cavity through a $12 \mathrm{~mm}$ trocar inserted in a central incision. A standard laparoscope was also inserted laterally to record the global intra-abdominal scene. The procedure was divided into three steps: general exploration, motion compensation testing, and micropositionning testing.

1) Exploration: The surgeon first carried out an exploration of the abdominal cavity. He was able, without any difficulty, to position the tip of the micropositioner normal to any organ surface. To reach some organs, a grasper was needed (e.g. to move the bowels, to lift the liver). To insert this additional instrument, a $5 \mathrm{~mm}$ trocar was placed laterally. 


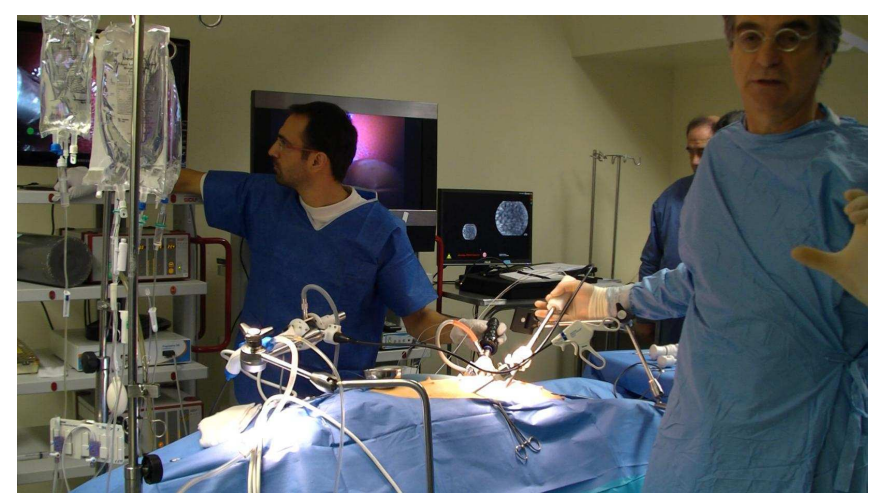

Fig. 11. The experimental setup for the in vivo tests

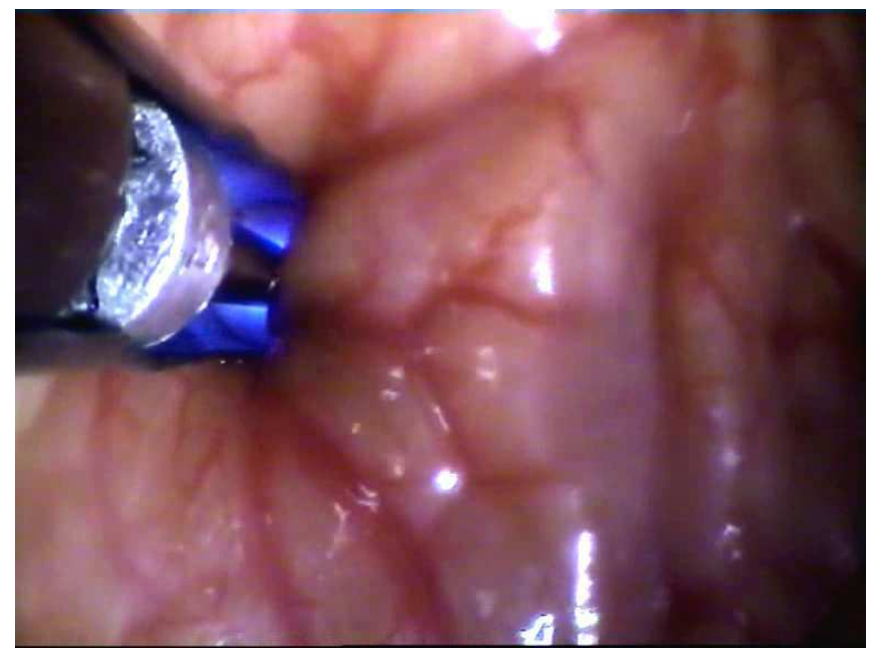

Fig. 12. Embedded endoscopic view of the system during the acquisition of the images that were used to compute the mosaic of Fig. 15. The tube of the stabilizer is cut on one side to allow taking video images

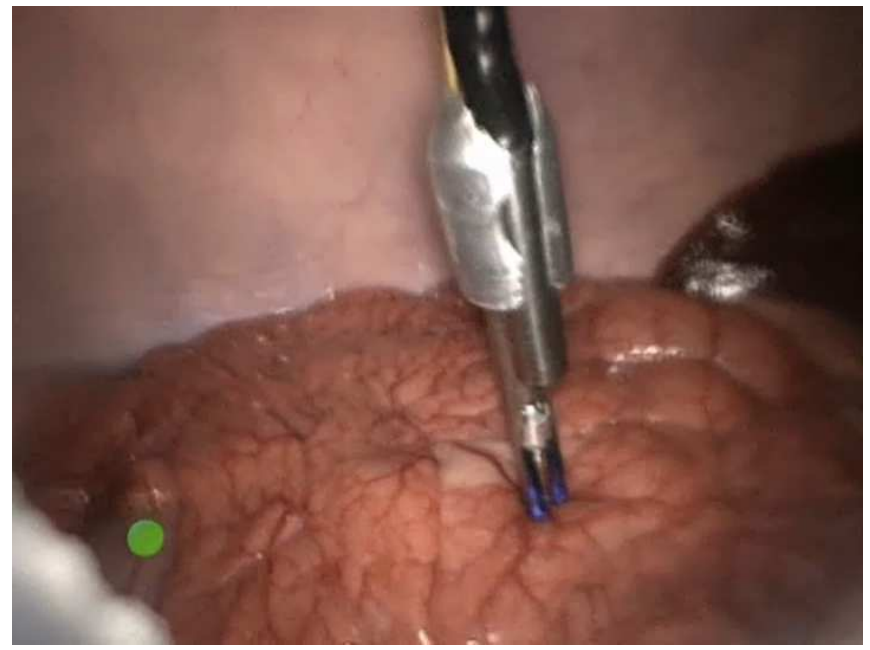

Fig. 13. General laparoscopic view of the system during the acquisition of a mosaic

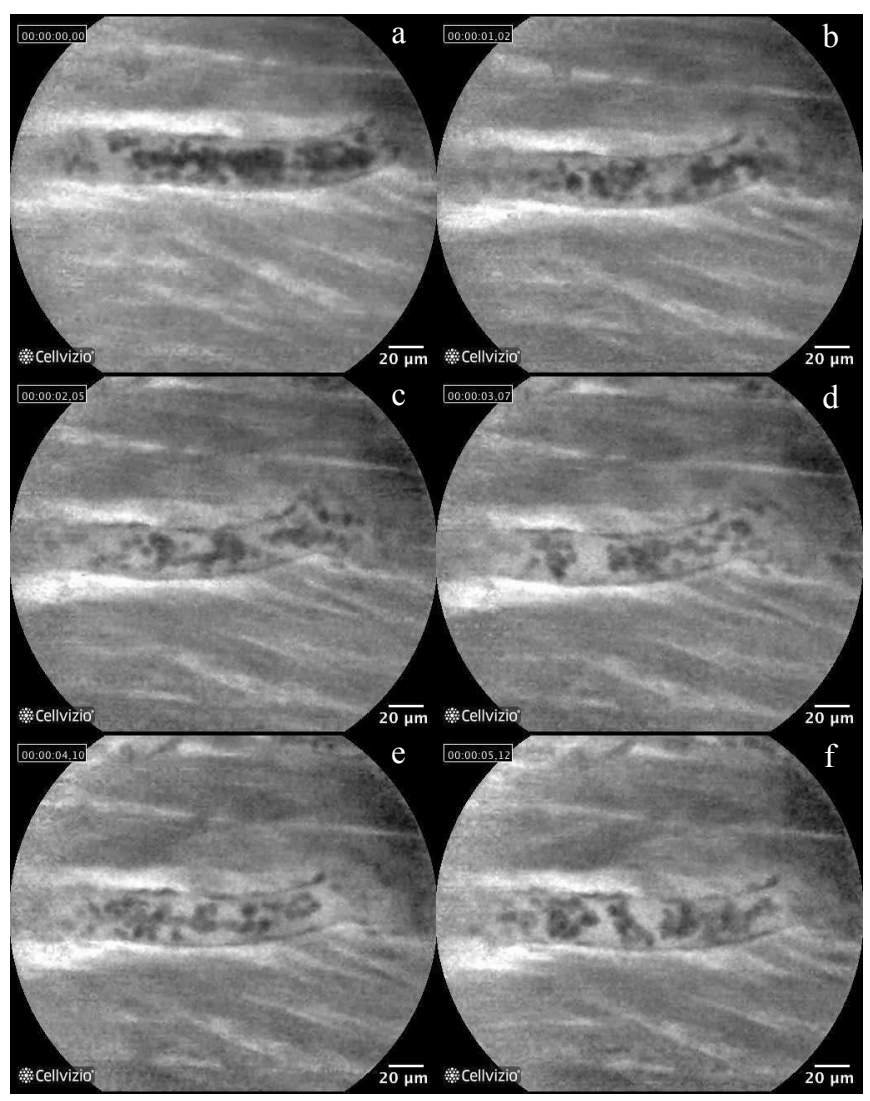

Fig. 14. Confocal images taken in vivo on the bowel of a porcine model with a Cellvizio UHD probe, with stabilization: a) $t_{0}$; b) $t_{0}+1.02 \mathrm{~s}$; c) $t_{0}+2.05 \mathrm{~s} ;$ d) $t_{0}+3.07 \mathrm{~s} ;$ e) $t_{0}+4.10 \mathrm{~s} ;$ f) $t_{0}+5.12 \mathrm{~s}$

2) Motion Compensation: The motion compensation was tested with the micropositioner unactuated. The three balloons were over-inflated, so that the probe was locked centrally inside the micropositioner. As the micropositioner is attached to the stabilizer, the probe was then immobile with respect to the stabilizer. The system was put in contact with several moving organs (e.g. liver, pancreas, bowel, spleen). Fig. 12 is the embedded endoscopic view of the micropositioner during acquisition on the bowel and Fig. 13 is the general laparsocopic view of the complete scene. Fig. 14 shows a series of microscopic images acquired on the bowel: the resulting motion amplitude does not exceed $20 \mu \mathrm{m}$. The images were stable over several breathing periods (images on Fig. 14 range over $5 \mathrm{~s}$ ). We should highlight the fact that the cylindrical stabilizer was cut for this experiment (on the right side, as seen in Fig. 15) in order to keep a direct view on the probe, without decreasing the stabilization performance.

3) Micropositioning: Actuating the probe gave also encouraging results. It was possible to control the direction and the speed of the probe movements accurately, despite the contact with the tissue and the large breathing motions. The mosaics computed gave us a good estimate of the probe movement along the tissue surface, as shown on Fig. 15. Starting from top-left, the probe center (red dots) followed a nearly straight line towards the right. The second part 


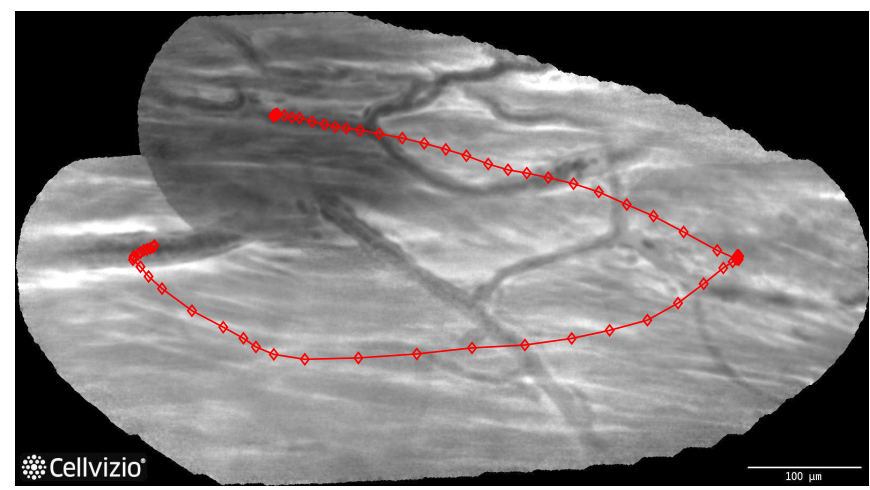

Fig. 15. Mosaic computed from in vivo images acquired on the bowel of a porcine model with a Cellvizio probe. The red dots are the centers of the successive images

of the motion was not straight, due to a contact between the balloons-bundle link (see Fig. 5) and the inner face of the stabilizer, resulting in a circular motion. Improving the design of the micropositioner will ensure a maximization of the probe workspace.

\section{CONClusions AND Future Work}

Traditional biopsy is an invasive and time consuming procedure. Hence, proceeding to optical biopsies with the help of probe-based confocal endomicroscopy could improve the surgical procedures of cancer therapy. In this paper, we proposed a macro-micro association to carry out large movements in the abdominal cavity for exploration, and micro-scale movements of a confocal probe for making mosaics on the organs, along with a passive mechanical physiological motion compensation.

Our passive mechanical physiological motion compensation method was tested during an in vivo trial on a porcine model. Residual motion was found to be less than $20 \mu \mathrm{m}$ during several breathing periods, which is very satisfactory in regard of the confocal probe field of view $(200 \times 240 \mu \mathrm{m})$.

The actuation principle, using hydraulic micro balloons mounted on a catheter, performed satisfactorily. The openloop control scheme gave good results in term of direction and speed control and allowed us to compute a mosaic from images taken during the in vivo trial. Further experiments will allow an optimization of our design parameters.

\section{ACKNOLEDGEMENTS}

This work was supported by OSEO (Maisons-Alfort, France) under ISI Project number I0911038W.

Many Thanks to Mauna Kea Technologies (Paris, France) and Endocotrol Medical (La Tronche, France) for their help and support.

\section{REFERENCES}

[1] T. Vercauteren, A. Perchant, G. Malandain, X. Pennec, and N. Ayache, "Robust mosaicing with correction of motion distortions and tissue deformations for in vivo fibered microscopy," Medical Image Analysis, vol. 10, no. 5, pp. 673-692, Oct. 2006.

[2] K. E. Loewke, D. B. Camarillo, W. Piyawattanametha, M. J. Mandella, C. H. Contag, S. Thrun, and J. K. Salisbury, "In vivo micro-image mosaicing," IEEE Trans. Biomed. Eng., vol. 58, no. 1, pp. 159-171, Jan. 2011.

[3] V. Becker, T. Vercauteren, C. H. von Weyhern, C. Prinz, R. M. Schmid, and A. Meining, "High-resolution miniprobe-based confocal microscopy in combination with video mosaicing," Gastrointestinal Endoscopy, vol. 66, no. 5, pp. 1001-1007, Nov. 2007.

[4] G. L. Goualher, A. Perchant, M. Genet, B. Viellerobe, B. Abrat, and N. Ayache, "Towards optical biopsies with an integrated fibered confocal fluorescence microscope," in Proc. IEEE International Conference on Medical Image Computing and Computer-Assisted Intervention (MICCAI'04), 2004, pp. 761-768.

[5] A. Polglase, "A fluorescence confocal endomicroscope for in vivo microscopy of the upper- and the lower-GI tract," Gastrointestinal Endoscopy, vol. 62, no. 5, 2005.

[6] A. R. Rouse, A. Kano, J. a Udovich, S. M. Kroto, and A. F. Gmitro, "Design and demonstration of a miniature catheter for a confocal microendoscope," Applied Optics, vol. 43, no. 31, pp. 5763-5771, Nov. 2004.

[7] K. Kwok, V. Vitiello, and G. Yang, "Control of articulated snake robot under dynamic active constraints," in Proc. IEEE International Conference on Medical Image Computing and Computer-Assisted Intervention (MICCAI'10), 2010, pp. 229-236.

[8] S. Lee, Y. Nakamura, K. Yamane, T. Toujo, S. Takahashi, Y. Tanikawa, and H. Takahashi, "Image stabilization for in vivo microscopy by highspeed visual feedback control," IEEE Trans. Robot., vol. 24, no. 1, pp. 45-54, 2008.

[9] L. Ott, F. Nageotte, P. Zanne, and M. D. Mathelin, "Simultaneous physiological motion cancellation and depth adaptation in flexible endoscopy," IEEE Trans. Biomed. Eng., vol. 56, no. 9, pp. 1-4, 2009.

[10] D. Noonan, C. Payne, J. Shang, V. Sauvage, R. Newton, D. Elson, A. Darzi, and G. Yang, "Force adaptive multi-spectral imaging with an articulated robotic endoscope," in Proc. IEEE International Conference on Medical Image Computing and Computer-Assisted Intervention (MICCAI'10), 2010, pp. 245-252.

[11] A. Ruzzu, K. Bade, J. Fahrenberg, and D. Maas, "Positioning system for catheter tips based on an active microvalve system," Journal of Micromechanics and Microengineering, vol. 8, no. 2, p. 161, 1998. 